\title{
Wie lange antikoagulieren bei Muskelvenenthrombose?
}

Frage: Wie lange muss bei einer Muskelvenenthrombose die Antikoagulation durchgeführt werden? Richtet sich die Dauer nach dem Vorhandensein von thrombotischem Material in der B-ModeSonographie? Wird somit bei Persistenz des thrombotischen Materials länger antikoaguliert?

Dr. Christoph Nielen, Mönchengladbach: In der Leitlinie wird hierzu folgendes geschrieben: „Die Muskelvenenthrombose betrifft häufiger die Soleusmuskelvenen als die Venen der beiden Gastrocnemiusmuskeln. Bei progredientem Wachstum in das tiefe Venensystem sind bei den Soleusmuskelvenen die posterioren Tibialisvenen oder die fibularen Leitvenen und bei den Gastrocnemiusmuskelvenen die Vena poplitea betroffen. Die Häufigkeit der Progression und die auslösenden Faktoren sind nicht bekannt, da valide Studien hierzu fehlen.

Nach Gillet beträgt die initiale Rate an symptomatischen Embolien bei einer Muskelvenenthrombose 7\% und die Rate an Rekanalisierungen unter therapeutischer Antikoagulation liegt bei 85\%. Nach Absetzen der Antikoagulation kommt es innerhalb von zwei Jahren in 18,8\% der Fälle zu Rezidivthrombosen und ist damit vergleichbar mit der Rezidivrate proximaler Thrombosen.

Ergebnisse einer Kohortenstudie legen nahe, dass das Einwachsen einer Muskelvenenthrombose in die Unterschenkelleitvenen durch eine 10-tägige Gabe von NM-Heparin bei paralleler Kompressionstherapie verhindert wer- den kann. Die ideale Dauer der Antikoagulation bei Muskelvenenthrombose ist unklar; sie richtet sich vielerorts nach den subjektiven Beschwerden und dem Ultraschallbefund.“

Mein persönlicher Rat wäre, je nach Lokalbefund, Ausdehnung - nur $2-3 \mathrm{~cm}$ oder $>10 \mathrm{~cm}$ - etc, sowie vorliegender Schmerzsymptomatik eine Antikoaguation für mindestens zehn Tage, bei Risikoprofil eventuell auch acht Wochen, aber immer zusammen mit einer konsequenten Kompression mit Strümpfen der Kompressionsklasse (CCL) 3.

Literatur siehe Leitlinie;

zu finden unter www.awmf.org/leitlinien

Expertenrat Angiologie/Phlebologie,

www.springermedizin.de

\section{Kommt Nasenbluten von Bluthochdruck?}

Frage: Wenn ich einen Patienten mit $\mathrm{Na}$ senbluten zum HNO-Arzt schicke, bekomme ich in der Regel die Empfehlung, den Blutdruck optimal einzustellen. Doch nicht selten besteht überhaupt keine behandlungsbedürftige arterielle Hypertonie. Besteht überhaupt ein Zusammenhang zwischen Nasenbluten und Hypertonie?

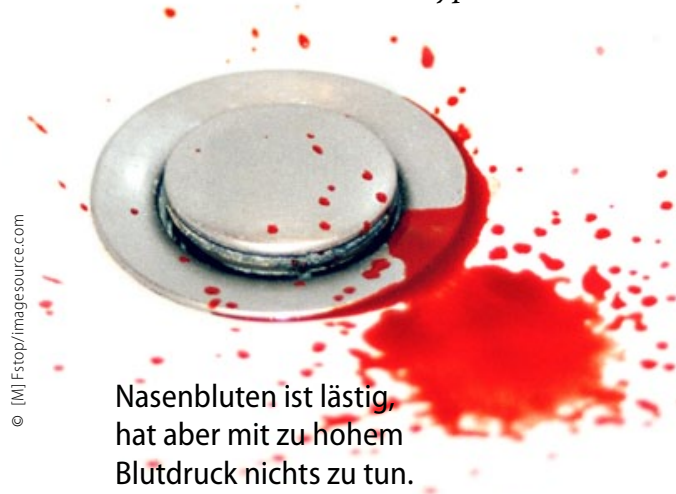

Dr. Peter Stiefelhagen, Hachenburg: Kommt es zu Nasenbluten, ist der Patient im Allgemeinen initial besorgt und verängstigt, sodass reflektorisch der Blutdruck etwas ansteigt. Dies bedeutet jedoch keinesfalls, dass eine behandlungsbedürftige arterielle Hypertonie vorliegt. Dass ein kausaler Zusammenhang zwischen Bluthochdruck und Nasenbluten vorliegt, gehört zu den hartnäckigsten Mythen in der Medizin; denn beim Nasenbluten handelt es sich um eine venöse

Blutung, die nichts mit dem Blutdruck zu tun hat. Ansonsten müsste ein erhöhter Blutdruck auch eine Hämorrhoidalblutung auslösen!

-Expertenrat Allgemeinmedizin, www.springermedizin.de
Osteoporose-Prophylaxe

\section{Ab wann zum DXA-Screening?}

Frage: Ab welchem Alter sollten Frauen zur Knochendichtemessung und in welchen Abständen? Welche Regel gilt für Männer?

Prof. Dr. Walter J. Faßbender, Zürich: Ein generelles Screening mittels DXAMessung ist nicht sinnvoll. Vielmehr sollte, gemäß DVO-Leitlinie, zunächst ein Risikoscreening durchgeführt werden. Die Kriterien hierzu sind der Leitlinie zu entnehmen. Eine klinische Untersuchung, dann ggf. auch mit DXA-Messung, ist bei Frauen $>70$ und bei Männern $>80$ Jahren sinnvoll. Spezielle Beachtung sollten Patienten mit hohem Osteoporose- und Frakturrisiko bei sekundären Formen der Osteoporose erhalten, z. B. Patienten, die unter einer Glukokortikoidtherapie stehen.

Expertenrat Osteoporose, www.springermedizin.de 